\title{
DRUG UTILIZATION PATTERN IN AN INTENSIVE CARE UNIT AT A TERTIARY CARE TEACHING HOSPITAL IN OMAN
}

\author{
Al-ZAKWANI I ${ }^{1}$, Al-THUHLI M ${ }^{2}$, Al-HASHIM A ${ }^{3}$, Al BALUSHI KA ${ }^{1 *}$ \\ ${ }^{1}$ Department of Pharmacology \& Clinical Pharmacy, College of Medicine \& Health Sciences, Sultan Qaboos University, Al-Khod, Oman. \\ ${ }^{2}$ College of Medicine \& Health Sciences, Sultan Qaboos University, Al-Khod, Oman. ${ }^{3}$ Department of Anesthesia and Intensive Care, \\ Sultan Qaboos University Hospital, Al-Khod, Oman. Email: mme51@squ.edu.om
}

Received: 11 September 2016; Revised and Accepted: 26 October 2016

ABSTRACT

Objectives: The objective of this study was to evaluate the drug prescribing trends in the intensive care unit (ICU) and correlate them with the disease patterns and patients' outcomes.

Methods: The case records of patients admitted to the ICU at Sultan Qaboos University Hospital, a teaching hospital in Oman, during a 5-month period between June $1^{\text {st }}$ and October $31^{\text {st }} 2013$ were analyzed. The variables collected included demographic and clinical characteristics as well as duration of hospitalization, indications, and utilization of different drugs.

Results: A total of 138 patients were evaluated with an average age of $46 \pm 19$ years. The average length of stay at ICU was $4.5 \pm 3.5$ days. The average number of drugs per prescription was $8.0 \pm 4.6$. The most common route used was the parenteral route (66\%) followed by the enteral route (25\%). Anti-infective drugs were the most commonly prescribed class of drugs (25\%) followed by gastrointestinal drugs (20\%). Antibiotics accounted for $12 \%$ of the total drugs prescribed. Trauma was the most common indication for the admission to the ICU (22\%).

Conclusion: Drugs prescription patterns in the ICU appeared to be similar to those reported in previous studies. However, prescription protocols need to be addressed to guide appropriate use of drugs in the ICU setting.

Keywords: Drug utilization, Critical care, Pattern, Oman.

(C) 2017 The Authors. Published by Innovare Academic Sciences Pvt Ltd. This is an open access article under the CC BY license (http://creativecommons. org/licenses/by/4. 0/) DOI: http://dx.doi.org/10.22159/ajpcr.2017.v10i2.15148

\section{INTRODUCTION}

Problems associated with drug prescription are not uncommon worldwide. Prescribing errors are considered as a key threatening factor that affects patient safety throughout hospital practice. The main problems are medication errors and adverse drug events [1]. A metaanalysis of 35 studies between 1990 and 2005 indicated that medication errors occurred at an average rate of $5.7 \%$ of all drug administration episodes while adverse drug events affected, on average, 6.1 patients per 100 hospitalized [1]. A set of core drug use indicators has been compiled by the World Health Organization (WHO), which are useful for studying patterns of drug prescribing in health-care facilities. These indicators include average number of drugs per encounter, percentage of drugs prescribed by generic name, percentage of encounters with an antibiotic, percentage of encounters with an injection, and percentage of drugs prescribed from the essential drug list [2].

The intensive care unit (ICU) is a setting where most patients with serious diseases are admitted. Therefore, a large number of drugs are employed to save these patients and that make them susceptible to medication errors and adverse drug reactions [3]. According to the comparison held by Weber et al., 38\% of the total drug costs are accounted for the ICU and these costs have increased at greater rate against non-ICU drug costs [4]. Furthermore, the cost of hospitalization and drug treatment in the ICU are also high [5]

Since patients admitted to the ICU receive multiple drugs, the majority of these drugs are empirically prescribed and mainly based on physician previous experience resulting in the lack of quantitative precision of drugs usage [6]. For example, some European reviews have indicated that over $50 \%$ of all neonatal ICU patients received off-label or an unlicensed prescription [7]. Several studies correlated multiple factors with mortality and duration of hospitalization in the ICU. Bobek et al. showed that the number of medication classes was a significant independent factor associated with the length of stay in the ICU [8]. A study by Hartmann et al. reported that antibiotic therapy correlates with hospital mortality in patients staying for more than $24 \mathrm{hrs}$ in a surgical ICU [9].

Even with the presence of drug use guidelines and policies in the ICU, it might be difficult to apply them due to the patient-specific disease state and physician medication preferences [8]. To improve therapeutic practices in such a case, it was found that the application of simple techniques of drug utilization was useful and inexpensive [10]. Therefore, utilization trends and costs of drugs prescribed in the ICU need to be urgently addressed.

Currently, there are no local data on drug prescribing trends of doctors at the ICU of Sultan Qaboos University Hospital (SQUH), a tertiary care teaching hospital in Oman. The aim of the current study was to evaluate the drug utilization pattern and prescribing trends in the ICU at SQUH and correlate them with disease patterns and patients' clinical outcomes. Studying drug prescribing pattern in the ICU is critically important in guiding prescription practices, and therefore, will help in ensuring an optimal outcome and containing costs.

\section{METHODS}

\section{Design and setting}

The study was conducted in the ICU at SQUH, a tertiary care hospital in the Sultanate of Oman. At SQUH, the ICU is part of the Department of Anesthesia, Intensive Care and Pain Management. The department has 20 adult beds that include coronary care unit also. 
This was a retrospective cross-sectional study of the case records of all patients admitted to the ICU at SQUH during the period from $1^{\text {st }}$ June 2013 to $31^{\text {st }}$ October 2013. Patients who stayed for a long-term ( $>20$ days) and patients who stayed $<24 \mathrm{hrs}$ in the ICU were excluded from the study.

\section{Data collection}

The collected patients' data included demographics (name, gender, age and weight), differential diagnoses on admission, duration of hospitalization in the ICU, final diagnoses, and patient's clinical outcome. Medication prescription details were also collected including number of drugs prescribed on the day of admission, administered doses, route of administration, and class/name of medications prescribed for each patient.

Ethical approval was obtained from the Medical Research and Ethics Committee of the College of Medicine and Health Sciences at Sultan Qaboos University. This approval provides permission to utilize patients' data in a confidential manner without disclosing patients' details.

\section{Statistics}

Descriptive statistics were used to describe the data. For categorical variables, frequencies and percentages were reported. For continuous variables, the means and standard deviations were used to summarize the data. The analyses were performed using Pearson's Chi-square tests, Student's t-test, and Mann-Whitney test whenever appropriate. Statistical significance was fixed at the level of $\mathrm{p}<0$. Analyses were performed using Stata (Stata Corp LP, College Station, TX, USA).

\section{RESULTS}

\section{Patients characteristics}

This study analyzed 138 patient admissions with a total of 1098 prescriptions. The average age of the patients was $46 \pm 19$ years. The most frequent age was $21-40$ years group $(37 \%, n=51)$, with males representing $64 \%(n=88)$ of all patients. The mean of duration of stay in the ICU was $4.5 \pm 3.5$ days. The median drug treatment duration of the patients was 7.0 days (Table 1 ).

As shown in Table 2, the WHO core drug prescribing indicators included the average number of drugs per prescription was $8.0 \pm 4.6$, the percentage of injectable drug use was $66 \%$, and the percentage of antibiotics use was $12 \%$. In addition, out of 160 drugs, 77 (48\%) belonged to the WHO essential drug list.

\section{Drug prescribing indicators}

A total of 160 drugs (1098 prescriptions) were prescribed during the period of stay in the ICU. The average number of drugs prescribed per patient was $8.0 \pm 4.6$. The parenteral route was the most commonly prescribed route of drug administration (66\%) followed by enteral route $(26 \%)$ and then inhalation which accounted for $3.3 \%$ of the total drug prescriptions $(\mathrm{N}=1098)$.

Table 3 shows a list of the top common prescribed drugs. Omeprazole was the most frequently prescribed medication $(n=74,6.7 \%)$ followed by enoxaparin $(n=67,6.1 \%)$, paracetamol $(n=51,4.6 \%)$, ranitidine, tramadol $(n=38,3.5 \%)$, tazocin $(n=35,3.2 \%)$, and meropenem $(n=33$, $3.0 \%)$.

As shown in Fig. 1 below, anti-infective drugs were the most commonly administered classes of drugs (25\%) followed by gastrointestinal drugs (20\%), medicine affecting the blood (10\%), and cardiovascular drugs $(8.8 \%)$

\section{Disease pattern and patients' clinical outcome}

The diagnoses of patients admitted to the ICU were further categorized and are shown in Fig. 2. The majority of patients had trauma (22\%) followed by cardiovascular (13\%), gastrointestinal system's diseases
Table 1: Demographic/clinical characteristics of patients during ICU stay

\begin{tabular}{ll}
\hline Clinical characteristics & Parameter \\
\hline Total number of patients $(\mathrm{N})$ & 138 \\
Age, mean \pm SD, (years) & $46 \pm 19$ \\
Age groups, years (n) & 7 \\
Up to 20 & 51 \\
$21-40$ & 43 \\
$41-60$ & 37 \\
More than 60 & \\
Sex n (\%) & $50(36 \%)$ \\
Female & $88(64 \%)$ \\
Male & $68 \pm 19$ \\
Weight*, mean $\pm S D$, kg & $4.5 \pm 3.5$ \\
Duration of stay at ICU, days (mean $\pm S D)$ & $8.0 \pm 4.6$ \\
Number of drugs prescribed/patient (mean $\pm S D)$ & 7 \\
Drug treatment duration, days (median) & \\
\hline
\end{tabular}

*Missing 26. SD: Standard deviation, ICU: Intensive care unit

Table 2: WHO core prescribing indicators $(\mathrm{N}=138)$

\begin{tabular}{ll}
\hline Indicator & Parameter \\
\hline Average number of drugs per encounter, mean \pm SD & $8.0 \pm 4.6$ \\
Percentage of encounters with an antibiotic & $12 \%$ \\
Percentage of encounters with an injection & $66 \%$ \\
Percentage of drugs prescribed from the essential & $48 \%$ \\
drug list & \\
\hline
\end{tabular}

drug list

SD: Standard deviation, WHO: World Health Organization

Table 3: List of the top common prescribed drugs in the intensive care unit ( $\mathrm{N}=1098$ prescriptions)

\begin{tabular}{lll}
\hline Medication & $\begin{array}{l}\text { Route of } \\
\text { administration }\end{array}$ & $\begin{array}{l}\text { Number of } \\
\text { prescriptions (\%) }\end{array}$ \\
\hline Omeprazole & Parenteral & $74(6.70)$ \\
Enoxaparin & Parenteral & $67(6.10)$ \\
Paracetamol & Enteral/parenteral & $51(4.60)$ \\
Ranitidine & Enteral/parenteral & $38(3.50)$ \\
Tramadol & Enteral/parenteral & $38(3.50)$ \\
Tazocin & Parenteral & $35(3.20)$ \\
Meropenem & Parenteral & $33(3.00)$ \\
Nonformulary drug & Parenteral & $32(2.90)$ \\
Ceftriaxone & Parenteral & $31(2.80)$ \\
Vancomycin & Parenteral & $24(2.20)$ \\
Potassium chloride & Enteral/parenteral & $24(2.20)$ \\
\hline
\end{tabular}

(13\%), and infections (8.0\%). Regarding the outcomes of patients during their stay in the ICU, about $75 \%$ of the patients were shifted to the general wards while $25 \%$ were deceased.

\section{Associations of drugs patterns and outcomes}

The difference in the clinical outcome with the total number of drugs prescribed per patient as well as the length of stay in the ICU was statistically significant ( $\mathrm{p}=0.023$ and 0.004 , respectively). No significant associations were noted between clinical outcome and age as well as between clinical outcome and gender $(p>0.05)$.

\section{DISCUSSION}

The present study evaluated drug utilization patterns in the ICU at SQUH. ICU is a setting where seriously ill patients who often suffer from multiple organ dysfunctions are administered. Therefore, the number of prescribed drugs and the cost of treatment and hospitalization are usually high [11]. Periodic studies on drug utilization are useful to meet the needs of assessing drug prescribing trends and optimizing pharmacotherapy. 


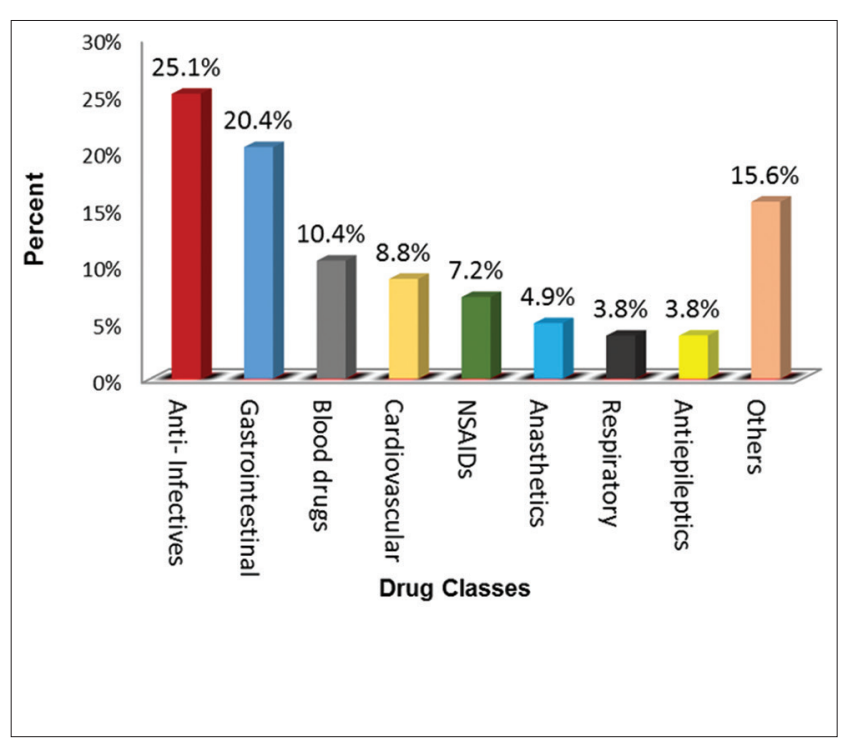

Fig. 1: Classes of drugs prescribed in the intensive care unit $(\mathrm{N}=1098$ prescriptions). NSAIDs: Non-steroidal anti-inflammatory drugs

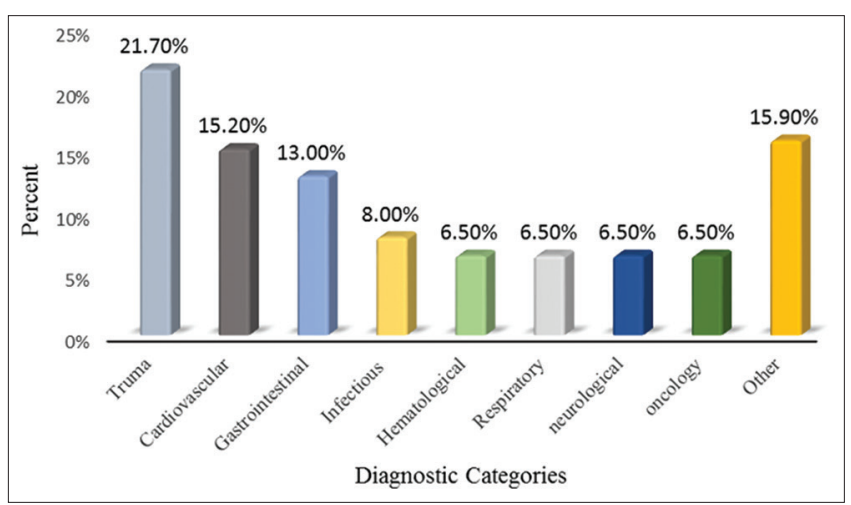

Fig. 2: Diagnostic categories of patients in the intensive care unit ( $\mathrm{N}=138$ patients)

The demographic data of 138 patients admitted to the ICU during the five months study period revealed that the mean age of the patients was $46 \pm 19$ years, with males representing $64 \%$ of the patients. This finding was similar to the two studies conducted in eastern and southern India in 2007 and 2011 [12-14]. Another study by Smythe et al. documented equal number of male and female individuals admitted to the ICU; however, with a significantly higher average age of 65 years [15]. Previous Iranian studies have reported that male patients accounted for more cases than female patients $(68 \%$ and $32 \%$, respectively) [16].

The most frequent age group was 21-40 years. This was in contrast to Shankar et al. study, which reported $>49$ years as the most frequent age group [5]. The mean length of stay of all patients admitted to the ICU was 4.5 days. This was similar to a retrospective study conducted in Canada, which showed that the average duration of ICU stay was 4.7 days [3]. This number was significantly low compared with American and Iranian studies with 9.4 and 6.0 days, respectively $[10,16]$. Therefore, early discharge from ICU plays a major role in minimizing economic burden.

A total of 1098 prescriptions for 138 patients were included in the study. The average number of drugs per patient (encounter) was 8.0 with a maximum number of 22 drugs per patient. This is lower or comparable to other previous studies. In an American study conducted in a trauma ICU, the average number of prescribed drugs was 9.1 [17] while an Indian study showed an average of 13.5 drugs [11]. A study in a French medical ICU reported that the average number of prescribed drugs was 5.0 [18]. By optimizing the administration of drugs within the ICU, the risk of drug-drug interactions and development of antibiotics resistance are minimized [19]. Therefore, it is important to keep number of prescribed drug as low as possible.

The WHO core indicators of prescribing practices measure the performance of health-care providers in several key dimensions related to the appropriate use of drugs. In our study, the percentage of antibiotics use was $12 \%$ and the percentage of injectable drug use was $66 \%$. About $52 \%$ of drugs were prescribed from the WHO essential drug list. This finding was similar to the study from America that showed $50 \%$ of prescribed drugs used in the ICU either belong to the WHO essential drug list or complementary drug list [10].

The most frequent route of drugs administration found in the current study was the parenteral route (66\%) followed by enteral route (26\%). Intravenous administration accounted for the majority of the parenteral drugs. Results of other studies carried out in India and Nepal also documented that parenteral drugs were the most frequent routes of administration $(87 \%, 51.12 \%$ and $53 \%$, respectively) $[5,11,20]$. In contrast, Paudel et al. reported that oral tablets (48\%) were the most commonly used dosage form in the ICU followed by injection and intravenous fluid (33\% and $7.8 \%$, respectively) [19]. Since patients admitted to the ICU suffer from severe diseases and need rapid interventions, parenteral drugs were found to be the most frequently prescribed which provide immediate onset of action. This might be a possible explanation for our finding.

The most commonly prescribed drug was omeprazole ( $\mathrm{n}=74 ; 6.7 \%)$, followed by enoxaparin $(n=67 ; 6.1 \%)$ and paracetamol $(n=51 ; 4.6 \%)$. A study in Western Nepal found that pantoprazole was the most frequently prescribed [19]. Among the ten most prescribed medicines, ranitidine was the most prescribed in another previous study [11]. Omeprazole is significantly important in suppressing gastric acid secretion, prevention of upper gastrointestinal bleeding, and promotion of mucosal healing. The present finding is sensible since endoscopic studies observed that between $75 \%$ and $100 \%$ of ICU patients had stress-related mucosal disease within $24 \mathrm{hrs}$ of admission. As a result, administration of acid suppression therapy (e.g., omeprazole) for stress ulcer prophylaxis in the ICU is well recommended to prevent gastrointestinal bleeding [21]. Omeprazole and enoxaparin are prescribed to all intubated patients unless contraindicated to prevent gastrointestinal bleeding and deep vein thrombosis, respectively.

According to the classification of the WHO Model List of Essential Medicines, anti-infective agents were the most commonly administered class of drugs $(25 \%)$. Within the anti-infective class, tazocin was the most prescribed drug. This was similar to a study performed in Iran in 2010 [16]. However, an Indian study observed that anti-infective agents were the second most prescribed class among the top five dug classes in the ICU [13]. In view of the long period of stay and unconscious status of ICU patients, they are more prone to hospital-acquired infections which affect about 30\% of ICU patients [22]. Singh et al. documented that $20-30 \%$ of nosocomial infections in the hospital were contributed by the ICU [23]. This might be one of the probable reasons for the increase in the number of prescribed anti-infective agents.

The current study reported that trauma was the most common indication for the admission to the ICU (22\%) followed by cardiovascular diseases, gastrointestinal diseases, and infections $(15 \%$, $13 \%$ and $8.0 \%$, respectively). As mentioned earlier, patients admitted to the ICU at SQUH were younger compared with another study [11]. Consequently, the risk of having traffic car accidents is higher than other older age groups. This might be one reason for the increase in the number of traumatic conditions managed in the ICU. In contrast, two previous publications found that cardiovascular conditions were 
the most frequently encountered in the ICU $[11,19]$. The contrast may be explained by the fact that the majority of cardiovascular cases are managed in a special cardiac care unit at SQUH.

Our results of the most frequent indications are corresponding with the common classes of drugs prescribed. Among 138 patients admitted to the ICU during the study period, $103(75 \%)$ patients improved and were shifted to the general wards while $35(25 \%)$ patients died. The present findings agreed with those reported in a prospective observational study where the mortality rate was accounted for $28 \%$ of the total patients admitted [13]. Since ICU patients have an emergency illness with serious complication and undergo multiple emergent interventions, they are vulnerable to experiencing adverse outcomes. However, our ICU patients appeared to have a higher mortality rate compared with two studies carried out in 2005 and 2011 (15\% and $17 \%$, respectively) $[5,19]$. The current study demonstrated significant association between the total number of prescribed drugs and mortality as deceased patients received larger number of drugs $(p=0.023)$. This was consistent with the findings documented in another study [16]. In contrast to other reports in the literature, the present study found no significant association between mortality with patients' age and sex [24]. Moreover, the probability of death in our ICU was higher when duration of stay was long $(\mathrm{p}=0.004)$.

The ICU has a high prevalence of nosocomial infection and antibiotic resistance, resulting in an increasing number of administered antibiotics [22]. The current study found that antibiotics accounted for $12 \%$ of the total drugs prescribed. Tazocin was the most commonly prescribed agent among this class while metronidazole was reported as the most commonly prescribed agent in another study [19]. In comparison to previous reports conducted in Turkey and Nepal, this study demonstrated low rate of antibiotic exposure $30 \%$ and $58 \%$, respectively) [5,25]. Certain factors may contribute to the low prescription of antibiotics. Since the number of trauma patients admitted in our ICU was the highest, these patients were less likely to have comorbid complications that require antibiotics [3]. The mean duration of hospital stay in all patients was 4.5 days. This reduced the chances of exposure to the hospital environment and to the patients with infectious disease. However, further studies are needed to evaluate bacterial resistance trends in the ICU at SQUH.

Our study had its limitations. Being a retrospective study, the quality of data was dependent on the available records and the missing data were very common. The sample size is relatively small which may not fully reflect the accurate population characteristics. Moreover, assessment of financial burden of drugs consumption in the ICU was not addressed in our study.

\section{CONCLUSION}

In conclusion, the results of this audit evaluated the drug utilization trends in the ICU at SQUH. They appeared to be reasonable and similar to those reported in other previous studies. This study will provide a database for further researches that will determine the cost and patterns of drug use in the ICU. Prescription protocols need to be addressed to guide for appropriate use of drugs in the ICU setting.

\section{REFERENCES}

1. Krähenbühl-Melcher A, Schlienger R, Lampert M, Haschke M, Drewe J, Krähenbühl S. Drug-related problems in hospitals: A review of the recent literature. Drug Saf 2007;30(5):379-407.

2. World Health Organization. Introduction to Drug Utilization Research. $1^{\text {st }}$ ed. Geneva: World Health Organization; 2003.

3. Yamashita SK, Louie M, Simor AE, Rachlis A. Microbiological surveillance and parenteral antibiotic use in a critical care unit. Can J Infect Dis 2000;11(2):107-11.

4. Weber RJ, Kane SL, Oriolo VA, Saul M, Skledar SJ, Dasta JF. Impact of intensive care unit (ICU) drug use on hospital costs: A descriptive analysis, with recommendations for optimizing ICU pharmacotherapy. Crit Care Med 2003;31 1 Suppl: S17-24.

5. Shankar PR, Partha P, Dubey AK, Mishra P, Deshpande VY. Intensive care unit drug utilization in a teaching hospital in Nepal. Kathmandu Univ Med J (KUMJ) 2005;3(2):130-7.

6. Williams A, Mathai AS, Phillips AS. Antibiotic prescription patterns at admission into a tertiary level intensive care unit in Northern India. J Pharm Bioallied Sci 2011;3(4):531-6.

7. Kumar P, Walker JK, Hurt KM, Bennett KM, Grosshans N, Fotis MA. Medication use in the neonatal intensive care unit: Current patterns and off-label use of parenteral medications. J Pediatr 2008;152(3):412-5.

8. Bobek M, Hoffman-Hogg L, Bair N, Mion L, Arroliga A, Slomka J. Utilization patterns, relative costs, and length of stay following adoption of MICU sedation guidelines. Formulary 2001;36(9):664-73.

9. Hartmann B, Junger A, Brammen D, Röhrig R, Klasen J, Quinzio L, et al. Review of antibiotic drug use in a surgical ICU: Management with a patient data management system for additional outcome analysis in patients staying more than 24 hours. Clin Ther 2004;26(6):915-24

10. Biswal S, Mishra P, Malhotra S, Puri GD, Pandhi P. Drug utilization pattern in the intensive care unit of a tertiary care hospital. J Clin Pharmacol 2006;46(8):945-51.

11. Patel MK, Barvaliya MJ, Patel TK, Tripathi C. Drug utilization pattern in critical care unit in a tertiary care teaching hospital in India. Int J Crit Illn Inj Sci 2013;3(4):250-5.

12. Chatterjee S, Mandal A, Lyle N, Mukherjee S, Singh AK. Drug utilization study in a neonatology unit of a tertiary care hospital in Eastern India. Pharmacoepidemiol Drug Saf 2007;16(10):1141-5.

13. John L, Devi P, John J, Guido S. Drug utilization study of antimicrobial agents in medical intensive care unit of a tertiary care hospital. Asian J Pharm Clin Res 2011;4(2):81-4.

14. Abideen S, Vivekandan K, Mishra P. Assessment of prevalence of potential drug-drug interactions in medical intensive care unit of a tertiary care hospital in India. Asian J Pharm Clin Res 2015;8(1):125-30.

15. Smythe MA, Melendy S, Jahns B, Dmuchowski C. An exploratory analysis of medication utilization in a medical intensive care unit. Crit Care Med 1993;21(9):1319-23.

16. Tavallaee M, Fahimi F, Kiani S. Drug-use patterns in an intensive care unit of a hospital in Iran: An observational prospective study. Int $\mathrm{J}$ Pharm Pract 2010;18(6):370-6.

17. Boucher BA, Kuhl DA, Coffey BC, Fabian TC. Drug use in a trauma intensive-care unit. Am J Hosp Pharm 1990;47(4):805-10.

18. Bonmarchand G, Czernichow P, Chrétien P, Massari P, Lecomte F, Hantute N, et al. Drugs used in a medical intensive care unit. Ann Fr Anesth Reanim 1986;5(5):497-501.

19. Paudel R, Palaian S, Giri B, Hom K, Sah A, Poudel A. Clinical profile and drug utilization pattern in an intensive care unit of a teaching hospital in Western Nepal. Arch Pharm Pract 2011;2(4):163-9.

20. Balaji V, Aithal S, Geetha S, Swetha ES. Drug utilization pattern among geriatric patients admitted in medical intensive care unit of a tertiary care teaching hospital. Asian J Pharm Clin Res 2015;8(2):281-3.

21. ASHP Therapeutic Guidelines on Stress Ulcer Prophylaxis. ASHP Commission on Therapeutics and approved by the ASHP Board of Directors on November 14, 1998. Am J Health Syst Pharm 1999;56(4):347-79.

22. Vincent JL. Nosocomial infections in adult intensive-care units. Lancet 2003;361(9374):2068-77.

23. Singh N, Yu VL. Rational empiric antibiotic prescription in the ICU. Chest 2000;117(5):1496-9.

24. Fowler RA, Sabur N, Li P, Juurlink DN, Pinto R, Hladunewich MA, et al. Sex-and age-based differences in the delivery and outcomes of critical care. CMAJ 2007;177(12):1513-9.

25. Usluer G, Ozgunes I, Leblebicioglu H; Turkish Antibiotic Utilization Study Group. A multicenter point-prevalence study: Antimicrobial prescription frequencies in hospitalized patients in Turkey. Ann Clin Microbiol Antimicrob 2005;4(16):16 